\title{
Nachhaltigkeitsprüfung in Deutschland weiterentwicklen
}

\author{
In Deutschland wird zunehmend über die \\ Institutionalisierung einer systematischen \\ Folgenabschätzung von Gesetzen im Hinblick \\ auf Nachhaltigkeit diskutiert. Die Erfahrungen \\ in anderen europäischen Ländern zeigen, dass \\ die ausgewählten Faktoren für die Funktions- \\ fähigkeit dieses Instruments ausschlaggebend \\ sind. \\ Von Klaus Jacob und Julia Hertin
}

D as Bundeskabinett hat Ende Mai beschlossen, die gemeinsame Geschäftsordnung der Bundesregierung so zu verändern, dass Nachhaltigkeitsaspekte ein obligatorischer Teil der Gesetzesfolgenabschätzung werden. Was ist von einer solchen Nachhaltigkeitsprüfung (NHP) zu erwarten? Wie kann diese konkret ausgestaltet werden? Welche Erfahrungen gibt es dazu bereits in anderen Ländern? Im Rahmen einer Studie, die von der Freien Universität Berlin im Auftrag und gemeinsam mit der Bertelsmann Stiftung erarbeitet wurde, wurden diese Fragen untersucht (Jacob et al. 2009). Ziel war es, Optionen zu erarbeiten, mit denen eine NHP in Deutschland zugleich dem politischen System angemessen und realistisch, aber auch anspruchsvoll ausgestaltet werden kann.

\section{Die Vorreiter von \\ Nachhaltigkeitsprüfungen in Europa}

Dazu wurde eine Analyse der Praxis in anderen Ländern durchgeführt, die eine solche NHP bereits eingeführt haben, beziehungsweise Nachhaltigkeitsaspekte in der allgemeinen Folgenabschätzung integriert haben. In Europa sind dies Belgien, Großbritannien, Irland, die Schweiz und die Europäische Kommission. Die Einführung beziehungsweise eine grundsätzliche Reform der Systeme liegt oft erst kurze Zeit zurück. Die längste Erfahrung hat Großbritannien seit der Einführung 1986 gesammelt, wobei das Verfahren 2007 grundsätzlich reformiert wurde. Dabei wurde das Verfahren der Folgenabschätzung wieder stärker auf wirtschaftliche Aspekte fokussiert und Nachhaltigkeitsaspekte wurden in einem Prüfmodul zusammengefasst, das nur bei vermuteter Relevanz angewendet wird. Die Europäische Kommission praktiziert ihr integriertes Impact Assessment seit 2003, die Schweiz hat 2004 eine separate Nachhaltigkeitsprüfung eingeführt, Irland integrierte Nachhaltigkeitsaspekte in die allgemeine Folgenabschätzung im Jahr 2005 und Belgien führte 2007 einen speziellen Nachhaltigkeitstest ein. In einigen Ländern ist die NHP demnach als separates Modul oder eigenständiger Prüfschritt vorgesehen, während sie in anderen Ländern in die allgemeine Gesetzesfolgenabschätzung integriert wurde.

Die Zusammenarbeit zwischen den Ressorts sowie mit nichtstaatlichen Akteuren unterscheidet sich ebenfalls. Eine intensive Beteiligung ist beispielsweise in der Kommission, in Großbritannien und Irland vorgesehen, während in Belgien die Prüfung normalerweise ohne externe Beteiligung durchgeführt wird. In allen Systemen sind verschiedene Prozessschritte wie die Aufteilung in Vor- und Hauptprüfung vorgesehen. Die Prüfbereiche und -kriterien sind unterschiedlich detailliert. Teilweise gibt es umfassende Fragen- und Checklisten, teilweise wird die Kosten-Nutzen-Analyse favorisiert, teilweise ist die Methodenwahl den Bearbeitern freigestellt.

Die Ergebnisse werden in Großbritannien und der Kommission umfassend veröffentlicht, in Irland sind die Resultate der Folgenabschätzungen wenigstens zum Teil öffentlich, dagegen sehen die Verfahren in der Schweiz und in Belgien keine Veröffentlichung vor. Eine Qualitätskontrolle findet in der Kommission durch das Impact Assessment Board, in Großbritannien durch die Better Regulation Executive, die im Wirtschaftsministerium angesiedelt ist, und in Irland durch die Better Regulation Unit, die beim Regierungschef angesiedelt ist, statt. Außerdem wurde in der Studie der Grad der Umsetzung erhoben. In der Kommission und in Großbritannien sind Gesetzesfolgenabschätzungen Routine, allerdings erfolgt die Prüfung von Nachhaltigkeitsaspekten nicht durchgängig und seit der Reform im Jahr 2007 scheint sich die Situation in Großbritannien verschlechtert zu haben. In Belgien und der Schweiz ist das Verfahren noch nicht über die Erprobungsphase hinaus gekommen. In Irland werden bisher nur die Vorprüfungen durchgeführt, zu vertiefenden Hauptprüfungen ist es hinsichtlich von Nachhaltigkeitsaspekten bisher noch nicht gekommen.

Bei aller Vielfalt der Systeme zeigt sich, dass nur wenige Jurisdiktionen überhaupt Erfahrungen mit der Berücksichtigung von Nachhaltigkeitsaspekten in der Gesetzesfolgenabschätzung haben. Die praktische Umsetzung und Integration in die Gesetzesvorbereitung ist anspruchsvoll. Die Beschreibung von Verfahren und die Bereitstellung von unterstützenden Materialien sind zwar sinnvoll, reichen aber nicht aus. Wichtig sind eine hochrangige politische Unterstützung und der Aufbau von administrativen Kapazitäten, um zu vermeiden, dass Prüfpflichten minimalistisch abgehakt werden. Weitere Erfolgsfaktoren sind Möglichkeiten und Anreize für eine interministerielle $\rightarrow$ 
Zusammenarbeit zu geben, Transparenz bei der Darstellung der Ergebnisse herzustellen und eine unabhängige Qualitätskontrolle einzuführen.

\section{Gestaltungsoptionen für Deutschland}

Welche Schlussfolgerungen können daraus für Deutschland gezogen werden? Ein separates Modul einer NHP, wie es auch bei der Bürokratiekostenschätzung in Deutschland praktiziert wird, hat die Vorteile, dass es möglicherweise eine höhere politische Aufmerksamkeit gewinnen kann und eine separate Qualitätskontrolle eher möglich ist. Allerdings besteht auch die Gefahr einer Konkurrenz und Überlappung mit anderen Prüfverfahren. Kooperation über Ressortgrenzen hinweg scheint in Deutschland besonders schwierig umsetzbar zu sein, zum einen wegen der großen Unabhängigkeit der Ministerien und zum anderen wegen politischer Konkurrenz in Koalitionsregierungen.

Eine kooperative, beteiligungsorientierte NHP würde einen Wandel in der Praxis der Politikvorbereitung darstellen, der nicht über Nacht zu erreichen ist. Eine rein interne Prüfung ist zwar mit einem geringeren organisatorischen Aufwand verbunden, dafür würde sie auf die Expertise externer Akteure verzichten.

Wann sollte eine NHP stattfinden? Je früher Nachhaltigkeitsaspekte mitgedacht werden, desto eher wird das einen Einfluss entfalten. Allerdings stehen zu einem frühen Zeitpunkt der Entscheidungsvorbereitung viele Eckpunkte noch nicht fest und eine Folgenabschätzung scheint kaum möglich. Ideal ist eine NHP, wenn sie den Prozess der Gesetzesvorbereitung begleitet und an verschiedenen Punkten unterstützt, so zum Beispiel die Vorprüfung zum Eckpunktepapier, die Hauptprüfung zum Referentenentwurf, die Diskussion der Ergebnisse in der interministeriellen Abstimmung und den öffentlichen Konsultationen sowie die Bewertung der Ergebnisse im Kabinett und im parlamentarischen Verfahren.

Welche Kriterien sollten geprüft werden? Das umfassende Konzept nachhaltiger Entwicklung bietet kaum Anhaltspunkte für eine Fokussierung auf bestimmte Teilaspekte. Eine Prioritätensetzung ist sachlich kaum zu begründen und bedarf der politischen Entscheidung und damit auch der Legitimation. Die Nachhaltigkeitsstrategie und die darin enthaltenen Ziele und Indikatoren bieten eine solche politisch abgestimmte Grundlage. Allerdings birgt der Verweis auf diese Auswahl von Indikatoren auch das Risiko, dass frühzeitig eine Verengung erfolgt und wichtige Teilaspekte, die sich aus dem Regelungsvorhaben ergeben, nicht zum Gegenstand gemacht werden.

Welche Methoden sollen genutzt werden? Eine Standardisierung der Methoden und insbesondere eine Quantifizierung bieten den Vorteil, dass verschiedene Optionen und Folgenkategorien miteinander verglichen werden können. Zudem ist eine Qualitätssicherung leichter möglich. Allerdings sind bei vielen Nachhaltigkeitsaspekten quantitative Daten nicht oder nicht mit vertretbarem Aufwand zu gewinnen. In der Praxis sind NHP meist qualitativ und beschreibend. Eine Methodenpluralität scheint dem Gegenstand sachgerechter zu sein.

Nicht zuletzt ist daher eine Qualitätskontrolle notwendig. Nur wenn eine Verbindlichkeit geschaffen wird und die in der NHP gewonnenen Erkenntnisse auch im weiteren Prozess zur Kenntnis genommen werden, gibt es ausreichende Anreize für die Ressorts und Referate, dieser auch ausreichend Aufmerksamkeit und Kapazitäten zuzuweisen. Eine Qualitätssicherung sollte die Einhaltung von Prozessstandards, insbesondere hinsichtlich der Beteiligung zum Gegenstand haben. Sie kann prüfen, ob die verschiedenen Nachhaltigkeitsaspekte ausreichend beleuchtet worden sind und kann beurteilen, ob die Methoden sachgerecht waren. Eine solche Funktion könnte, wie in Großbritannien, Irland oder der Kommission, im Kanzleramt angesiedelt werden oder durch ein externes Gremium erfolgen, wie das der Normenkontrollrat für die Bürokratiekostenschätzung leistet. Das Parlament und insbesondere der Parlamentarische Beirat könnte eine aktive Rolle bei der Bewertung der Ergebnisse der NHP übernehmen.

Ähnlich wie bei der Bürokratiekostenschätzung könnte eine Verankerung einer Nachhaltigkeitsprüfung als Gesetz vorteilhaft sein und eine größere Verbindlichkeit nach sich ziehen, vor allem wenn ein Gremium wie der Normenkontrollrat eine externe Qualitätssicherung betreibt.

\section{Schlüsselelemente einer anspruchsvollen Prüfung}

Die Vergleiche mit anderen Ländern und die Auswertung der Erfahrungen in Deutschland zeigen, dass eine anspruchsvolle Ausgestaltung insbesondere darauf zielen sollte, dass zum einen eine umfassende und möglichst frühzeitige interministerielle Zusammenarbeit ermöglicht werden sollte, und zum anderen mit Mechanismen für Transparenz und Qualitätskontrolle sichergestellt werden sollte, dass es auch eine Nachfrage nach den Ergebnissen gibt. Dann gäbe es stärkere Anreize, um die notwendigen administrativen Kapazitäten aufzubauen. Das würde sich aber auch lohnen, denn eine anspruchsvolle NHP kann, wenn sie nicht nur auf dem Papier existiert, sondern in der Praxis gelebt wird, ein starkes Instrument zur Integration von Nachhaltigkeitsaspekten und für eine stärker evidenzbasierte Politikformulierung sein.

\section{- AUTOREN + KONTAKT}

Dr. Klaus Jacob ist Forschungsdirektor der Forschungsstelle für Umweltpolitik an der Freien Universität Berlin.

Freie Universität Berlin, Forschungsstelle für Umweltpolitik, Ihnestraße 22, 14195 Berlin. E-Mail: jacob@zedat.fu-berlin.de

Julia Hertin arbeitet seit 2008 als wissenschaftliche Mitarbeiterin beim Sachverständigenrat für Umweltfragen.

Julia Hertin, Sachverständigenrat für Umweltfragen (SRU), Geschäftsstelle, Reichpietschufer 60, 10785 Berlin. Tel.: +4930 263696-111, E-Mail: julia.hertin@uba.de

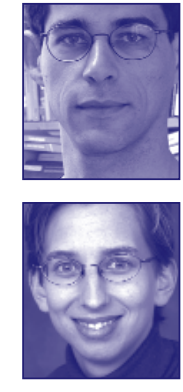


(c) 20I0 Authors; licensee IÖW and oekom verlag. This is an article distributed under the terms of the Creative Commons Attribution Non-Commercial No Derivates License (http://creativecommons.org/licenses/by-nc-nd/3.o/), which permits unrestricted use, distribution, and reproduction in any medium, provided the original work is properly cited. 